\title{
Development and pilot testing of a counseling-plus-mHealth intervention to reduce risk for pregnancy and sexually transmitted infection in young women with depression
}

\author{
Lydia A. Shrier ${ }^{1,2}$, Pamela J. Burke ${ }^{1,2,3}$, Sarah Parker ${ }^{1}$, Rori Edwards ${ }^{1}$, Cassandra Jonestrask ${ }^{1}$, \\ Emily Pluhar ${ }^{1}$, Sion Kim Harris,
}

${ }^{1}$ Division of Adolescent/Young Adult Medicine, Boston Children's Hospital, Boston, MA, USA; ${ }^{2}$ Department of Pediatrics, Harvard Medical School, Boston, MA, USA; ${ }^{3}$ School of Nursing, Bouvé College of Health Sciences, Northeastern University, Boston, MA, USA

Contributions: (I) Conception and design: LA Shrier, PJ Burke; (II) Administrative support: C Jonestrask; (III) Provision of study material or patients: LA Shrier; (IV) Collection and assembly of data: C Jonestrask; (V) Data analysis and interpretation: LA Shrier, PJ Burke, SK Harris; (VI) Manuscript writing: All authors; (VII) Final approval of manuscript: All authors.

Correspondence to: Lydia A. Shrier, MD, MPH. Division of Adolescent/Young Adult Medicine, Boston Children's Hospital, 300 Longwood Avenue, Boston, MA 02115, USA. Email: lydia.shrier@childrens.harvard.edu.

Background: Depressed young women have elevated rates of unintended pregnancy and sexually transmitted infections (STIs). The objective of this study was to develop and pilot-test a counseling-plusmHealth intervention to reduce sexual and reproductive health $(\mathrm{SRH})$ risk in young women with depressive symptoms.

Methods: Using the Behavior-Determinants-Intervention logic model, we developed the Momentary Affect Regulation-Safer Sex Intervention (MARSSI) to address the challenges that depression imposes on SRH risk reduction efforts of high-risk young women: (I) in-person counseling using motivational interviewing (MI) to elicit motivation for safer sex and develop a behavior change plan, and teaching cognitive-behavioral skills to manage negative thoughts and affective states; (II) 4-week Ecological Momentary Intervention (EMI) on a smartphone to report momentary phenomena related to depression and SRH risk, and receive personalized, tailored messages prompting healthy behaviors and encouraging cognitive-behavioral skill use when riskrelated cognitions and negative affect are reported; and (III) booster counseling to review behavior change goals and plans and teach a new cognitive-behavioral skill. We developed the counseling through iterative interviews with 11 participants and developed the EMI through a 2-week trial with three participants, then revised MARSSI to reflect participant feedback. We next conducted a pilot-test among depressed, highrisk female adolescent clinic patients age 15-24. Pilot participants completed mental health, motivation to change behavior, and SRH behavior assessments and provided feedback at baseline, post-EMI, and at 3-month follow-up. We analyzed participant retention, counseling duration, app engagement, intervention quality ratings, and participant feedback, and compared mental health and SRH risk behavior across the study.

Results: Seventeen participants completed the initial counseling session, 15 participated in the EMI, 14 returned for the booster session, and 14 completed the 3-month follow-up. App engagement was high for all 4 EMI weeks ( $\geq 1$ report/day for median $\geq 6$ days/week). Post-intervention, most or all participants agreed with each positive statement about the messages, reported "Excellent" MARSSI usefulness, and attributed improvements to MARSSI. Compared to baseline, post-EMI depressive symptoms, confidence to change self-selected risk behavior, and confidence to use the cognitive restructuring skill improved. At 3 months, depressive symptom scores remained lower and confidence to use cognitive restructuring remained higher, compared to baseline. Participants also reported lower frequency of sex, lower proportion of condomunprotected sex events, and, among those using effective contraception, more consistent condom use at 3-month follow-up vs. baseline.

Conclusions: MARSSI was feasible, acceptable, and engaging to young women with depression and 
SRH risk behavior, and was associated with increased confidence to reduce SRH risk, decreased SRH risk behaviors, increased confidence to use cognitive restructuring, and decreased depressive symptoms over 3 months. Future research is warranted to evaluate MARSSI's efficacy to improve motivation, skills, affect, and behaviors, as well as reproductive health outcomes in high-risk depressed young women.

Keywords: mHealth; sexual risk intervention; ecological momentary intervention; adolescent; young adult; women; motivational interviewing (MI); cognitive-behavioral skill

Received: 15 July 2019; Accepted: 18 November 2019; Published: 05 April 2020.

doi: 10.21037/mhealth.2019.11.05

View this article at: http://dx.doi.org/10.21037/mhealth.2019.11.05

\section{Introduction}

Adolescent and young adult (“young") women with depression have disproportionately high rates of adverse sexual and reproductive health (SRH) outcomes, including unintended pregnancy (1), unintended first birth (2), and sexually transmitted infections (STIs) $(3,4)$. Compared to non-depressed young women, young women with depression are more likely to engage in SRH risk behaviors such as no or inconsistent use of contraception (5), condom nonuse, sex with multiple partners, and sex under the influence of substances $(6,7)$. Depressed young women are also less likely to use long-acting reversible contraception (LARC) (1), which is more effective than oral contraceptives and non-hormonal methods (8). Several factors may contribute to increased SRH risk in depressed young women, including efforts to regulate feelings or emotions (affect) (9-14), impaired information processing and risk perception (15), substance-related sexual enhancement expectations (16) and substance use (17), unhealthy relationship dynamics (18-20), reproductive coercion (21), intimate partner violence (22), condom negotiation difficulties (20), and pregnancy ambivalence (23).

Although some pregnancy/STI interventions have recognized the particular needs of young women (24) and a few have been developed for depressed young people $(25,26)$, there have been no reports of pregnancy/STI prevention interventions designed specifically for depressed young women. Addressing factors related to emotional distress may enhance intervention effectiveness for depressed young women $(27,28)$. Depressed women may benefit at least as much from risk reduction interventions as nondepressed women $(29,30)$ and be more motivated to reduce their risk behaviors because they are invested in decreasing their distress (31). In addition, women with depression may experience decreased depressive symptoms during participation in a sexual risk reduction intervention $(27,32)$.

To date, SRH risk reduction interventions for young people, women, and individuals with depression have been primarily administered in settings where they come in contact with health care, such as clinics (33) and community health agencies (34), and where they gather for other purposes, such as schools (28). However, emotional and social experiences influence, and are influenced by, sexual decision-making and behaviors in the course of daily life and in natural life contexts $(12,14)$. Intervening at the time of these experiences may result in healthier decisionmaking. In addition, self-monitoring in real time and in real life can improve emotional self-awareness and decrease depressive symptoms, which can further decrease SRH risk (35).

Mobile technology offers a means of facilitating selfmonitoring and providing in-the-moment intervention to improve depressive symptoms and reduce SRH risk. Specifically, mobile technology can be used to provide realtime feedback on maladaptive thoughts and perceptions (cognitions) and unsafe behaviors, and prompting of adaptive strategies for affect regulation (attempting to alter feelings or emotions to improve experience) and coping (36). For example, depressed youth report low positive affect before sex (14) and negative affect before sex with a non-main partner (37). Mobile technology can prompt depressed young women to use strategies to recognize and respond to poor affect, and avoid undesired or unsafe sex. Ecological Momentary Interventions (EMIs) use mobile technology in this way to provide treatment in daily life (in real or near-real time and in natural settings) (38). With EMIs, treatment may be delivered in a variety of ways; users may receive push notifications, prompts to complete self-assessments and/or activities, and messages tailored to time, location, companionship, or other 
features of momentary context, as well as send messages and/or access web-based materials or live support via text message, chat, or phone. EMIs have been created for mood disorders (39), substance use $(36,40)$, and other health behaviors (38). Most young people in the U.S. $(\geq 95 \%)$ and other advanced economies, and a substantial and growing proportion of young people in emerging economies own or have access to a smartphone (41-43), making this approach to intervention particularly relevant for adolescents and young adults. For depressed young women in particular, their smartphone may be an important means of social connectivity and support (44). Young women with depression have indicated that an EMI to address associations between depressive symptoms and behavior would be acceptable, supportive, and helpful in reducing their SRH risk (45).

EMIs that combine mobile intervention with other approaches may result in longer-term effects than mobile intervention alone (46). Further, human counseling along with computer intervention may be more effective in changing adolescent behavior than solely computer intervention (47). Establishing an in-person relationship first may aid in the formation of a connection with a computer (48). When asked for their perspectives on an intervention for cannabis use that combined in-person counseling with an EMI, youth considered the mobile device an extension of the counselor and endowed it with human qualities such as caring, empathetic, and supportive (49). The objective of this research was to develop and pilot-test a counselingplus-mHealth intervention using EMI to reduce risk for pregnancy and STIs in adolescent and young adult women with depressive symptoms and high-risk sexual behavior.

\section{Methods}

We conducted a mixed-methods study of the intervention comprised of two stages: (I) development and refinement, and (II) pilot-testing. For both stages, we recruited primary care patients from two adolescent/young adult clinics affiliated with an urban children's hospital in the northeastern United States. One clinic, based at the hospital, draws primary care patients from the racially, ethnically, and socio-economically diverse surrounding urban and suburban communities; the other clinic draws patients from the local community in which it is situated, which has substantial representation of Hispanic/Latino ethnicity and low socio-economic status. We obtained institutional review board approval with a waiver of parental permission for participants under the age of 18 years. Clinic providers could identify patients as depressed and sexually active and offer a brochure about the study, or patients could self-refer by taking a brochure from a rack in the clinic. Interested patients could then meet with research staff for more information or provide their contact information for research staff to call them. Each participant met with a research staff member for a comprehensive informed consent process prior to any research activities. To aid with understanding, we used a video to illustrate study procedures.

\section{Intervention development and refinement}

\section{Design approach}

We used the Behavior-Determinant-Intervention Logic Model (50) to design the intervention (Figure 1). Our health goal is to reduce unintended pregnancy and STI among depressed adolescent and young adult women. We identified five behaviors affecting this goal, including using effective contraception, using condoms consistently and correctly, abstaining from sex, decreasing frequency of sex, and decreasing number of sex partners. We then identified important modifiable determinants of these behaviors that are particularly salient for young women with depression, based on review of literature, our prior research on depression and sexual risk behavior, and data from our interviews with depressed young women engaging in high-risk sexual behavior $(5,7,14,18,40,51-56)$. These determinants included low motivation to change behavior, negative affective states, maladaptive thoughts, low selfesteem, unhealthy relationships, poor communication skills, insufficient knowledge and poor planning skills. We then developed components and activities designed to modify these determinants.

\section{Intervention approach}

We chose a motivational interviewing (MI)-based approach to enhancing motivation to change behavior. Low motivation is a core symptom of depression that can interfere with expending effort despite potential reward (57). MI is a collaborative, non-judgmental, client-centered counseling style that is directed toward a specific behavior change goal (58). Enhancing low motivation to change SRH risk behaviors in a manner that is aligned with individual goals may contribute to effectiveness of pregnancy/STI prevention interventions $(59,60)$. We sought both to support individual goal identification and to develop a 


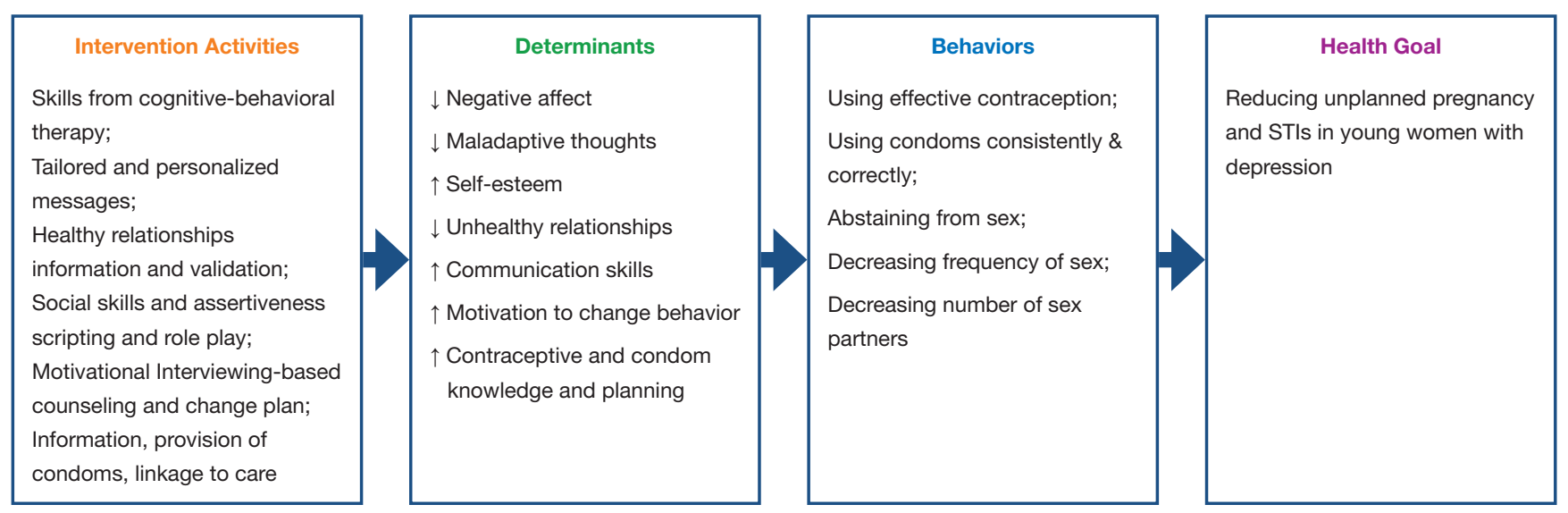

Figure 1 Behavior-Determinant-Intervention Model for the Momentary Affect Regulation-Safer Sex Intervention (MARSSI).

manualized approach to counseling that could be replicated. Therefore, based on formative research (56), we identified five areas of behavior change and developed a bubble sheet for agenda mapping (a tool to aid the counselor and the participant in collaboratively choosing a behavior change area to focus on in the session) (58). We grouped the five areas of behavior change into three broad topicsChoosing Not to Have Sex, Using Condoms Correctly and Consistently, or Using Effective Birth Controland created corresponding sections of the intervention manual, each guiding an MI-based discussion to facilitate behavior change planning. The behavior change discussion concluded with an activity to document a change plan using a game board as a visual aid (Figure 2 for an example). Each session also included discussion of healthy relationships, talking with a sexual partner, and brief conversations about the two behavior change topics not selected (to provide information and reinforce healthy behaviors, as applicable).

To address the affective and cognitive determinants, we drew on cognitive-behavioral therapy (CBT), an effective depression treatment in youth (61). CBT involves understanding the cognition-affect relationship, challenging distorted cognitions, developing effective problem-solving, using strategies to regulate affect and maladaptive behavior, and increasing behavioral activation such as engaging in enjoyable activities (62). We initially illustrated the cognition-affect-sexual behavior relationship through a series of brief scenarios that mapped onto each of five types of cognitive-behavioral skills: cognitive restructuring (identifying and challenging negative and unhelpful thoughts), social skills, activity scheduling, behavioral activation (increasing one's engagement in enjoyable activities), and problem solving. In the first draft of the intervention, we asked participants to read the scenarios and select one that best represented their experience and for which they wished to learn a skill. The counselor then led the participant through an activity to learn that cognitivebehavioral skill.

\section{Intervention development with participant collaboration}

To test the counseling session, we recruited female patients age 15-23 years with depressive symptoms [Patient Health Questionnaire (PHQ)-A or PHQ-9 score $\geq 8$ ] (63) who reported engaging in high-risk SRH risk behaviors in the past 3 months, including low effectiveness, inconsistent, or no contraception; inconsistent or no condom use, penileanal sex, more than one sexual partner, sex under the influence of alcohol or other drugs, or treated for an STI. One of the authors (LAS) conducted the counseling session in person or via secure video call, soliciting verbal feedback during and following the session. A co-author (CJ) observed the session and took field notes. The research team reviewed the feedback and notes, and revised the counseling manual accordingly. The counselor then met with each participant a second time to review the changes and solicit further feedback, with an emphasis on determining if her specific concerns, comments, and recommendations had been addressed. We offered participants $\$ 10$ in a gift card and payment for parking or public transportation for each of the two study visits.

We enrolled 11 young women with depressive symptoms and SRH risk. Mean age of participants was 21.0 years [standard deviation (SD) 2.0]. Most had moderate or 


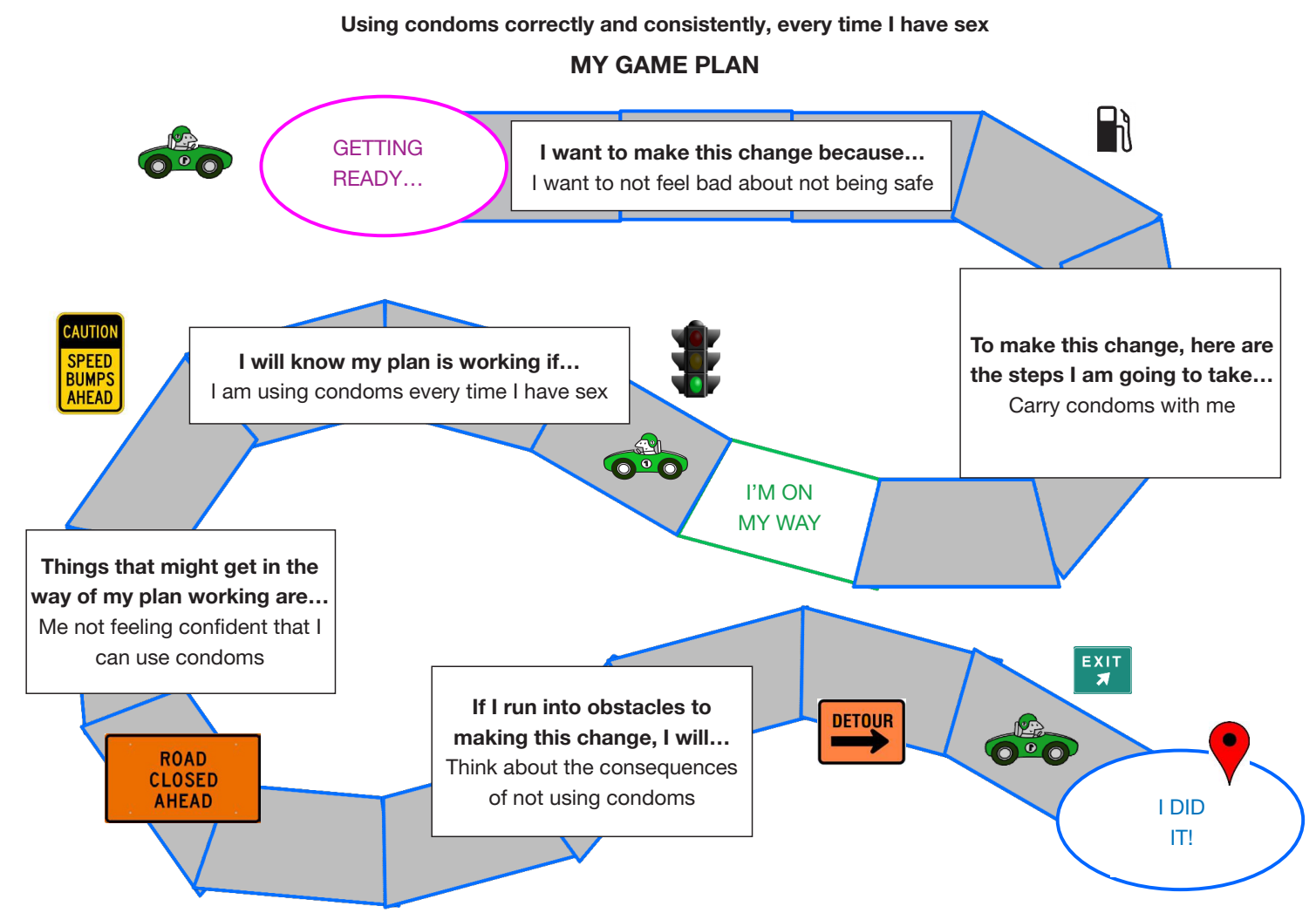

Figure 2 Example of completed My Game Plan in the Momentary Affect Regulation-Safer Sex Intervention (MARSSI).

moderate-severe depressive symptoms [PHQ score median 11 , interquartile range (IQR) 10-18] (63). The majority of participants $(72.7 \%)$ reported two or more SRH risk behaviors, most frequently not using condoms consistently $(72.7 \%)$ and using a low-effectiveness method as their primary form of contraception (63.6\%). All participated in one interview and $9(81.8 \%)$ participated in a second interview; one participant withdrew, indicating that she was engaged to be married and did not feel that she was a fit for the study, and one was lost-follow-up.

Participants were very positive about the counseling session. They indicated that the MI approach was collaborative, learning a cognitive-behavioral skill was a good idea, and the SRH content was appealing and useful. Participants identified areas for improvement, including session length (too long), cognitive-behavioral skills (some skills, such as behavioral activation, did not seem as relevant to SRH health as others, such as cognitive restructuring and social skills), and singularity of the session (preference for a second session).

We sought to enhance counseling effectiveness while conserving limited time, space, and personnel resources in clinics. Because the process of behavior change occurs in the natural environment, during the course of daily life, we developed an EMI (40) to facilitate self-monitoring of emotional or cognitive factors associated with SRH risk and deliver real-time messaging responsive to negative affect, maladaptive cognitions, and unsafe behaviors, offering support and prompting healthy strategies for affect regulation and coping. Based on our formative work $(45,56)$, we created message content and an algorithm to allow a young woman to choose message voice and style and to create her own messages, if she desired (Table 1). The app would be populated with pre-programmed messages according to the young woman's preferences and added any self-created content. We made several messages available for each voice and style to present varied content.

A young woman would download the app on her personal smartphone. The app would prompt her through a push notification to respond to brief surveys three times a day quasi-randomized within morning, afternoon, and evening time blocks (momentary reports) and once a day 
Table 1 EMI Messages in MARSSI

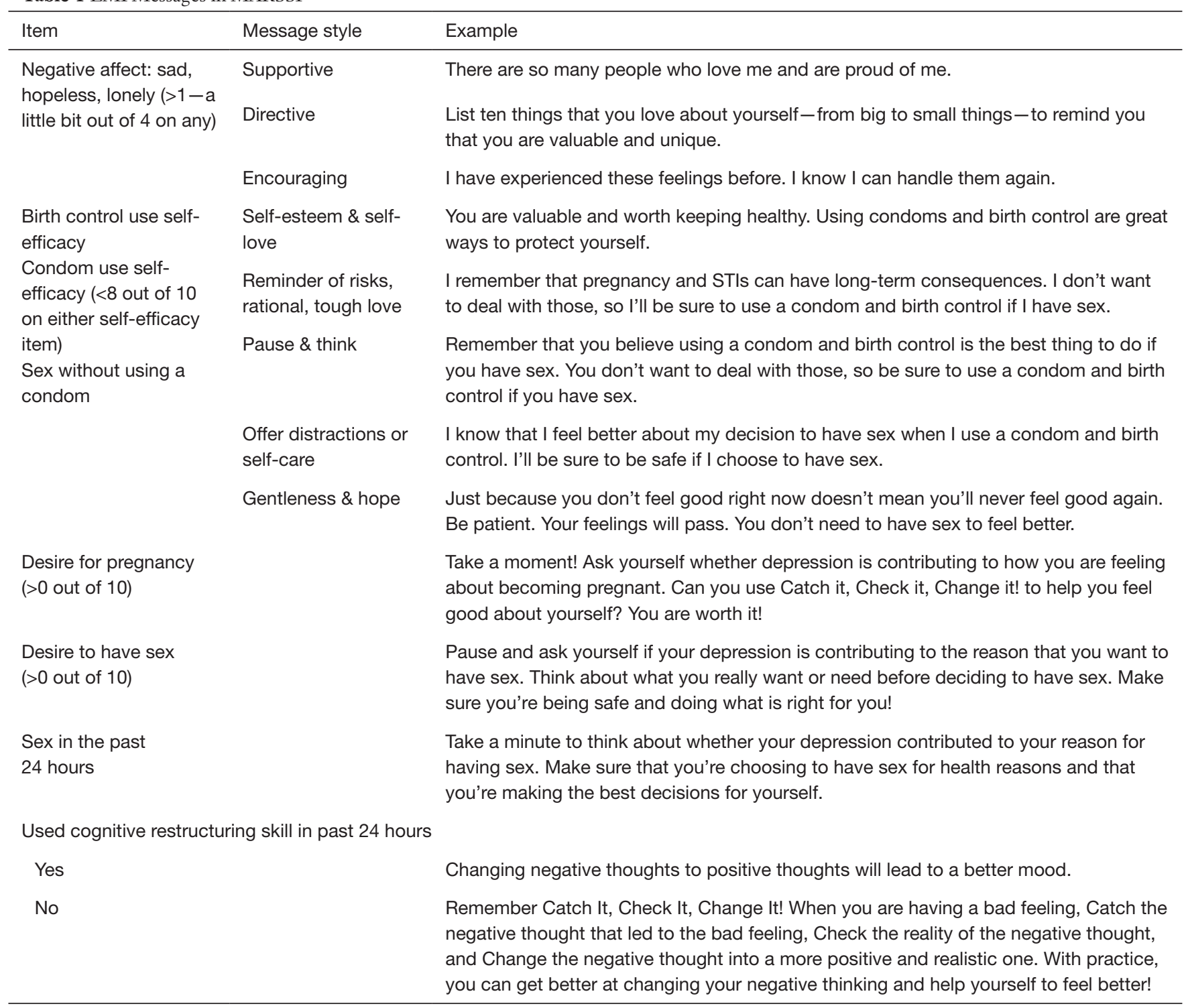

Participants also had the option to write their own message or upload a photo.

at a scheduled time (diary report). Survey items included questions on positive and negative affect, contraceptive selfefficacy, condom self-efficacy, desire for pregnancy, desire and reasons for sex, and sexual behaviors. Within each report, young women would receive her personalized messages tailored to responses indicating high negative affect, low contraceptive or condom self-efficacy, pregnancy desire, or desire for sex to regulate affect. Messages would appear automatically following specific responses. Young women would not be asked to acknowledge or reply to the messages.

We pre-tested the EMI component of the intervention with three young women who had participated in our formative interviews $(45,56)$ and had expressed an interest in continuing to collaborate with us on the research. In the development phase, participants completed surveys for 2 weeks, then provided feedback in an interview. Participants indicated that the EMI helped them to feel better, to use their cognitive-behavioral skill, and to be safer with their SRH behaviors. They rated the usefulness as "excellent" and indicated that the signal and message frequency was "the right number." They suggested that the EMI would be improved with greater message variety. 


\section{marssi圆}

Momentary Affect Regulation - Safer Sex Intervention

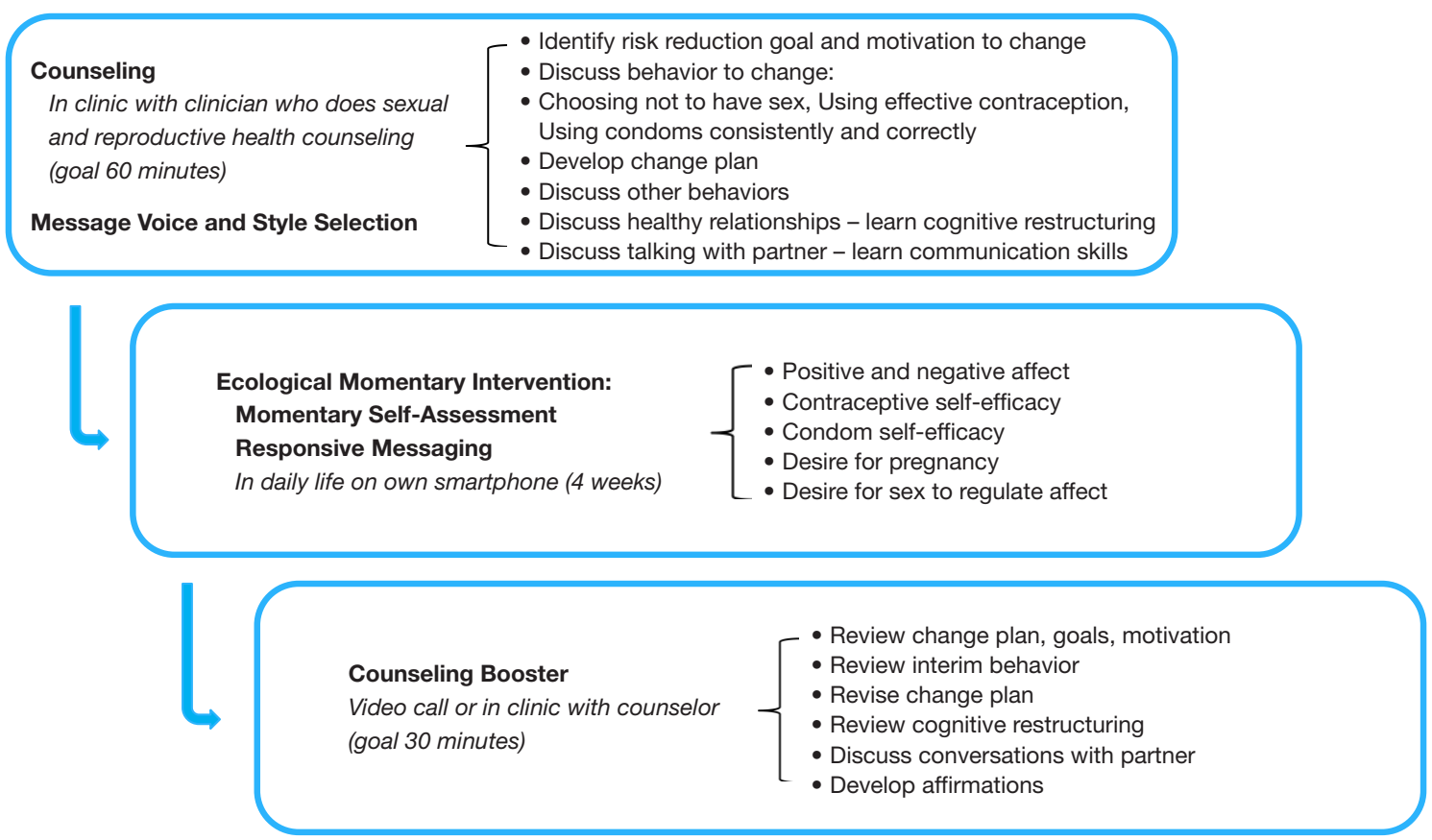

Figure 3 Momentary Affect Regulation-Safer Sex Intervention (MARSSI) overview.

\section{Intervention refinement}

Based on participant feedback and research team discussions, we made several changes to the intervention. We revised the eligibility criterion pertaining to depressive symptoms to PHQ score $\geq 5$ to align with published guidelines for at least mild depressive symptoms (63) and after observing that several patients considered themselves depressed or were considered by their clinician to be depressed, but had PHQ scores $<8$. We made the teaching of the cognitive restructuring and social skills activities more specific to how depression relates to sexual relationships and decision-making and eliminated teaching of the other three skills. We shortened the counseling session to take less than 90 minutes (ideally, duration of 60 minutes). We added a booster counseling session (duration of 30 minutes), after the EMI's to review the participant's change plan, goals, and motivation for change; discuss interval SRH behaviors; revise the change plan, if desired; review cognitive restructuring, if desired; replay and discuss conversations with her partner; and learn a new cognitive-behavioral skill (developing affirmations). We extended the EMI for a total of 4 weeks, expanded the message library and increased message variety. Finally, we simplified and standardized EMI message programming procedures. The revised MARSSI (Figure 3) was then tested in the pilot study.

\section{Pilot study}

\section{Procedures}

Patients were eligible if they were female; age 15-24 years; had a PHQ-9 score $\geq 5$; were having penile-vaginal sexual intercourse at least once a week, on average; reported at least one pregnancy/STI risk in the past 3 months (as described for the development cohort); and owned a smartphone. Based on our formative work, patients were excluded if they were pregnant (would not be using contraception), had given birth in the past 6 months (may have post-partum depression and, if parenting a newborn, particular difficulty adhering to the intervention schedule), or were married or engaged to be married (may have low motivation for using condoms for STI prevention).

Following informed consent/assent, participants selfcollected urine for pregnancy testing and a vaginal swab 
Table 2 Schedule of assessments

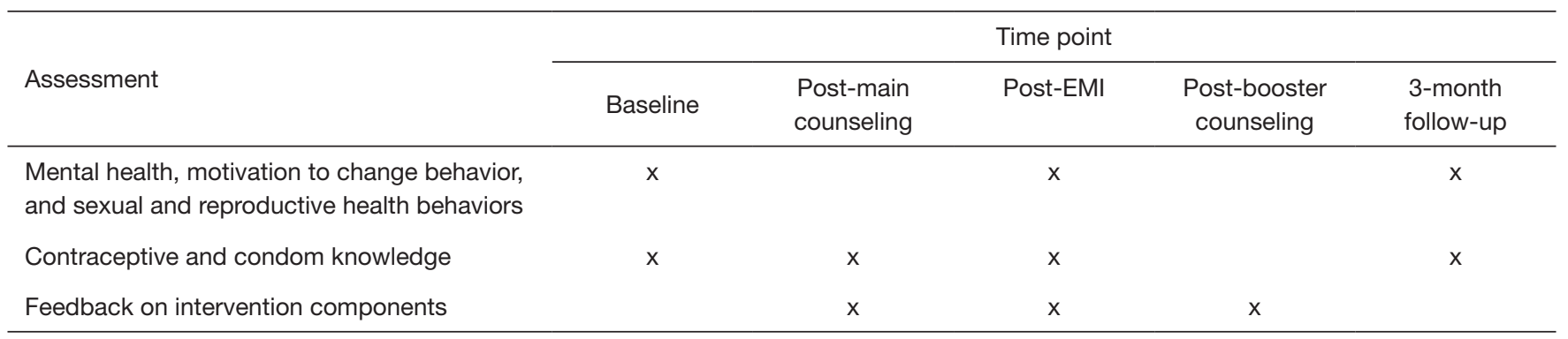

for testing for $N$. gonorrboeae and C. trachomatis. They then completed a baseline survey of mental health and SRH behaviors and a 28-day timeline follow-back (TLFB) calendar of penile-vaginal and penile-anal sexual intercourse events, partner type, contraception, condom use and problems during use, and substance use within 2 hours before sex (64) (see Table 2 for schedule of assessments). Participants put the study app (MetricWire, Inc.) on their personal smartphone and the research assistant trained them in its use. They were asked to complete one week of EMA reporting on their phone (3 momentary reports prompted at random within morning, afternoon, and evening intervals and 1 diary at a personally scheduled time each day). Participants then returned to the clinic for a scheduled meeting with a clinic staff member trained in MARSSI counseling (a nurse practitioner or nurse experienced in family planning counseling) and to personalize her EMI message style, voice, and content. After the counseling session, participants completed feedback and knowledge surveys. They then left the clinic and used the smartphone app for the 4-week EMI. At the end of the EMI, participants completed mood and behavior surveys and a 28-day TLFB. They then had a booster session with the counselor, by video call or in person (per their preference), followed by feedback surveys and a brief interview. After three months, we asked participants to complete mental health and behavior surveys, and a 28-day TLFB calendar. The research assistant contacted the participant via text message reminders about study activities. We offered pilot participants up to $\$ 100$ gift card remuneration (depending on completion of study activities) and payment for parking or public transportation associated with study visits.

\section{Measures}

\section{Sample characteristics}

On the baseline survey, participants provided date of birth, age, race, Hispanic/Latinx ethnicity, and education level.

\section{SRH risk}

We assessed STI treatment, number of sexual partners, condom use, use of a low-effectiveness method as the primary form of contraception (condoms, diaphragm, cervical cap, spermicide, sponge, fertility awareness, or withdrawal), penile-anal sex, and sex under the influence of alcohol or other drugs in the past 3 months.

\section{Feasibility}

We measured duration of the counseling sessions. We calculated the retention rate for the booster counseling session. We determined app engagement by calculating the number of days on which participants made at least one report in each week of the 4-week EMI (65). [We did not use response rate to the EMI prompts as a measure of app engagement. The intervention emphasizes choice and individual decision-making. Participants need to be able to choose whether to engage with the app during daily life. They may elect not to respond to a prompt if the prompt is delivered during an inconvenient time (e.g., while driving or in school); or if they do not want to consider their emotional state, cognitions, or risk behaviors; or if they do not want to receive messages.] To evaluate changes in contraceptive and condom knowledge covered in the counseling, we administered a 12-item true-false survey designed for this study at baseline, post-counseling, postintervention, and follow-up.

\section{Acceptability}

After the main counseling session, participants indicated agreement on a 5 -point Likert-type scale to 18 items on adherence to MI principles and perceived counseling utility (e.g., The counselor respected that any change will be up to me; My Game Plan helped me to think about what I want to change and how to do it) and assessed session quality overall (Poor, Fair, Good, Excellent) on one item. After the booster counseling session ("post-intervention"), participants responded to 11 similar MI and utility items for the booster 
session, as well as 4 Likert-type items on using the app (ease, question clarity, enjoyment, signal annoyance), one item on daily signal frequency (too few times, the right number of times, too many times), 8 Likert-type items on the messages (e.g., I liked the way the messages were written), and 11 Likerttype items on overall study comfort, burden, and utility (e.g., I have felt comfortable participating in this study, Taking part in this study has been burdensome, Taking part in this study has belped me to make the changes that I want to make). At 3-month follow-up, participants indicated agreement to 20 Likerttype items on study procedures (e.g., Sending the photo of the pregnancy test through the app was easy) and perceived utility (e.g., Taking part in this study helped me to be safer with my sexual behavior). Post-intervention and at 3-month followup, participants rated overall usefulness of the study (Poor, Fair, Good, Excellent), and completed free text on their most and least favorite parts of the study, what one thing they would change about the study, and any additional comments.

\section{Intervention individualization}

We evaluated how participants individualized MARSSI, including choice of behavior to change, choice of message style and voice, message creation, and choice of remote $v s$. in-person booster session.

\section{Changes in mental health, motivation to change behavior, and SRH behavior}

To prepare for a future efficacy trial, we developed measures for intermediate cognitive and emotional outcomes and targeted behavioral outcomes, and evaluated changes postEMI and at 3-month follow-up, compared to baseline. We examined PHQ-9 depressive symptoms score (range, 0-27) at baseline, post-intervention, and 3-month followup. To evaluate changes in motivation to change behavior, we evaluated confidence, readiness, and importance using $0-10$ rulers (58) at baseline, post-intervention, and 3 -month follow-up. To evaluate changes in use of cognitive restructuring as a means of managing depressive symptoms, we measured confidence to change negative thoughts to positive ones (0-10) from the Coping Self-Efficacy Scale (66) at baseline, post-intervention, and 3-month follow-up. On the EMI diary report, we asked whether in the past 24 hours the participant used the cognitive restructuring skill learned with the counselor (yes/no). To evaluate changes in SRH risk, we assessed sexual events (vaginal, anal), condom use (yes/no, broke, slipped), and partner type (regular, new, casual) on the 28-day TLFB at baseline, post-intervention, and follow-up.

\section{Analysis}

We computed descriptive statistics for all variables. We compared reporting days across the four EMI weeks using the related samples Friedman 2-way ANOVA rank test. We calculated for each time point (baseline, postEMI, 3-month follow-up) the sum score for the PHQ9 items, the 28-day frequency of sex, the proportion of events without a condom or for which the condom broke or slipped (condom unprotected sex events), and the proportion of events with a new or casual sex partner. Because of highly skewed data distributions, to evaluate changes from baseline to post-EMI, and from baseline to 3-months follow-up in mental health, motivation to change, and SRH risk, we used the Wilcoxon signedrank test for pairwise comparison (the non-parametric equivalent of a paired $t$-test) (67).

\section{Results}

\section{Participants}

We enrolled 21 young women in the pilot study, of whom 17 completed the baseline assessments (visit and EMA week) and returned for the counseling visit; these participants were able to contribute information regarding intervention feasibility and thus comprised the analytic sample. Although the numbers were too small to make statistical inferences about differences between those who did and did not return for the intervention, we did observe that participants who did not return for the intervention had lower levels of depressive symptoms and lower proportion of condom-unprotected sex events, compared to participants in the analytic sample (PHQ-9 score median 10, IQR 7.75-10.75 vs. median 15, IQR 9-19.5 and condom-unprotected sex events median $28.6 \%$, IQR 0-28.6\% vs. median 100\%, IQR 100-100\%, respectively).

Median age of the analytic sample was 20.6 years (IQR, 18.9-23.0 years). Just over one-half (58.8\%) of the sample self-identified as black/African-American race and $35.3 \%$ as white; $23.5 \%$ self-identified as Hispanic/Latinx ethnicity. Most $(88.2 \%)$ had completed high school. At baseline, median PHQ-9 depressive symptoms score was 15 (IQR, $9-19.5)$, in the moderately severe symptom range and consistent with a depressive disorder diagnosis $(63,68)$. Participants identified a variety of SRH risk behaviors in the past 3 months, including inconsistent or no condom use $(82.4 \%)$, sex under the influence of alcohol or other drugs 
Table 3 Feasibility and acceptability of Momentary Affect Regulation-Safer Sex Intervention (MARSSI) Counseling Sessions

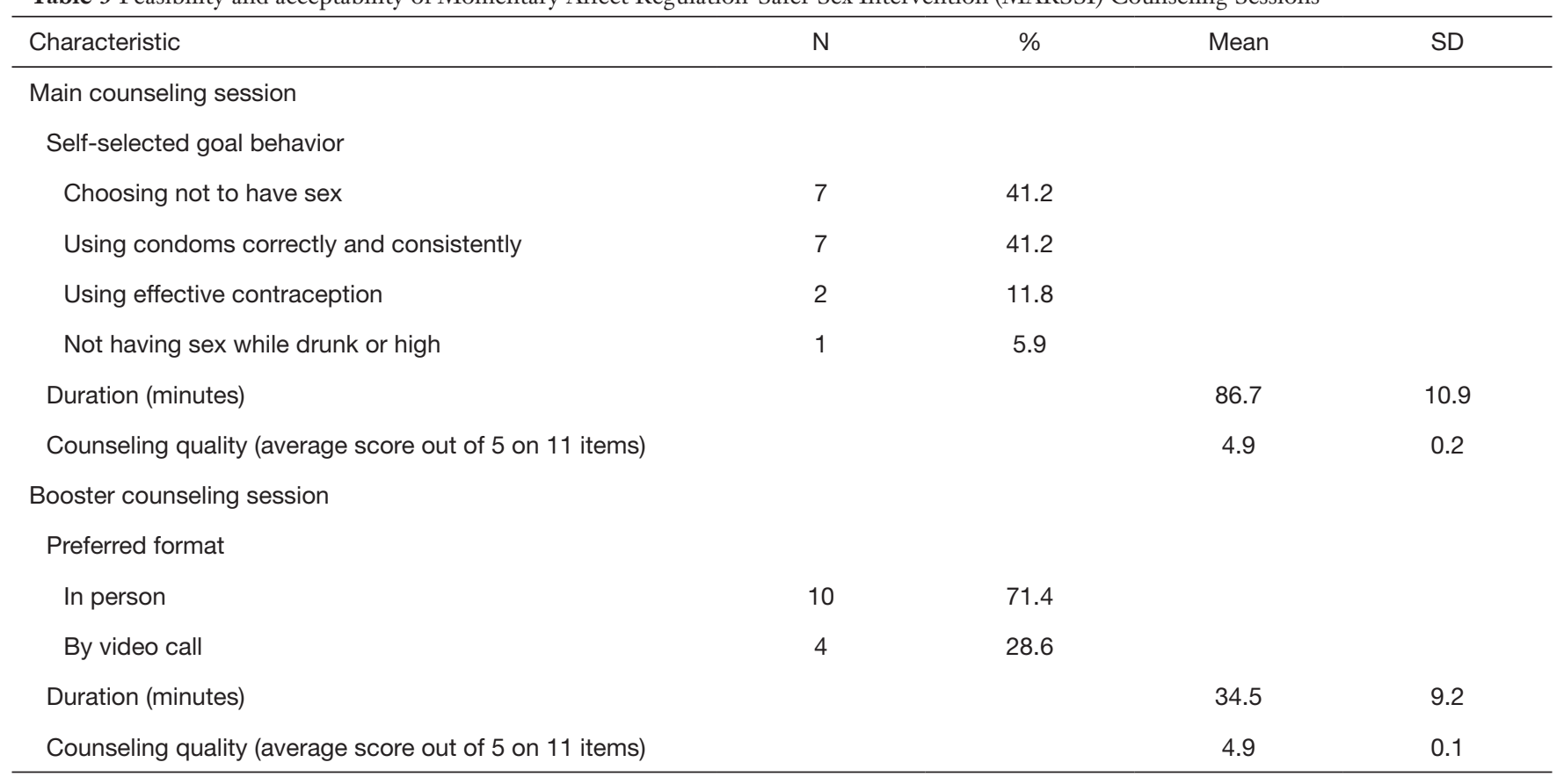

(58.8\%), more than one sexual partner (35.3\%), and lack of effective birth control (29.4\%); $87.5 \%$ of participants reported more than one SRH risk. One participant had a positive test for T. vaginalis at baseline and received prompt treatment according to standard clinical practice.

Of the 17 participants receiving the main counseling session, $17(100 \%)$ started and $15(88.2 \%)$ completed the EMI, 14 (82.4\%) completed the booster counseling session, and 14 (82.4\%) completed 3-month follow-up assessments. Participants who were $v s$. were not retained at follow-up did not differ with regard to mental health, motivation, or behaviors.

\section{Main and booster counseling session feasibility}

For their goal behavior, most participants (82.4\%) selected Choosing Not to Have Sex or Using Condoms Correctly and Consistently (Table 3). The session lasted a mean of 86.7 minutes (SD 10.9). Participants rated the counseling quality highly (mean item score 4.9 out $5, \mathrm{SD}$ $0.2)$. Fourteen participants $(82.4 \%$ of those starting the intervention) completed the booster counseling session; most $(71.4 \%)$ chose to complete the session in person (Table 3). The booster session lasted a mean of 34.5 minutes (SD 9.2). Counseling quality ratings for the booster session were highly favorable (mean item score 4.9 out 5 , SD 0.1 ).
Although contraceptive and condom knowledge was high on pretest (median 10 out of 12, IQR 10-11), no participant scored $100 \%$. However, on posttest, 9 participants $(52.9 \%)$ responded correctly to all 12 items. Condom and contraceptive knowledge scores remained high at the booster session and on 3-month follow-up (median 11 out of 12, IQR 11-12, for both).

\section{Message voice and style choices}

For the EMI messages following report of low positive or high negative affect, participants were nearly evenly split in choice of voice $\left(52.9 \%\right.$ chose $1^{\text {st }}$ person, $42.1 \%$ chose $2^{\text {nd }}$ person); a majority $(58.8 \%)$ chose the Encouraging style. For the messages following report of SRH risk cognitions or behavior, participants were again split in choice of voice $(42.1 \%$ chose $1^{\text {st }}$ person, $52.9 \%$ chose $2^{\text {nd }}$ person); just over one-half $(52.9 \%)$ chose the Gentleness \& Hope style. For both message types, at least one participant chose each of the available styles; none preferred to write her own message or upload a photo.

\section{EMI feasibility}

App engagement was high through the EMI period, declining modestly across the 4 weeks. Participants completed $\geq 1$ momentary report on a median of 7 out of 7 days (IQR, 
Table 4 Acceptability of Momentary Affect Regulation-Safer Sex Intervention (MARSSI) Ecological Momentary Intervention (EMI)

\begin{tabular}{lcc}
\hline Characteristic & N & $\%$ \\
\hline App acceptability (\% agree) & 14 & 93.3 \\
I enjoyed using the smartphone & 15 & 100 \\
App was easy to use & 15 & 100 \\
Questions were clear and understandable & 2 & 13.3 \\
I was annoyed by the signals & & \\
Message acceptability (\% agree) & 15 & 100 \\
I read the messages & 14 & 93.3 \\
I liked the way the messages were written & 15 & 100 \\
Messages helped me to feel better & 15 & 100 \\
Messages helped me to make the & & \\
changes I wanted to make & 14 & 93.3 \\
Messages helped me to use the skill I & & \\
learned with the counselor & 11 & 73.3 \\
Messages helped me to be safer & 3 & 20 \\
I received too many messages & 2 & 13.3 \\
I was annoyed by the messages & &
\end{tabular}

4.5-7.0 days) in week 1, 6.5 days (IQR, 3.3-7.0 days) in week 2, 6.0 (IQR, 4.3-7.0) days in week 3, and 5.0 (IQR, 3.0-7.0) days in week 4 (Friedman two-way ANOVA by ranks test statistic for comparison of median reports across weeks $10.94, \mathrm{P}=0.012$ ). One participant had no momentary reporting after week 1 , and one had no momentary reporting after week 3. Daily diary reports were completed a median of 5 of 7 days (IQR, 3-7 days) in week 1, 4.5 days (IQR, 4-6 days), and 4 days (IQR, 2-6 days) in both weeks 3 and 4 (Friedman two-way ANOVA by ranks test statistic $10.74, \mathrm{P}=0.013)$. One participant did not complete any diary reports after week 1 , one after week 2 , and one after week 3 . Overall, fifteen participants (88.2\%) completed at least one EMI report in each week for all four weeks; one participant did not complete any reports after the $1^{\text {st }}$ week and one participant did not complete any reports in the $4^{\text {th }}$ week. Sixteen participants $(94.1 \%)$ reported using the cognitive behavioral skill on at least one day during the EMI (median 7 days, IQR 2-17; median $77.8 \%$ of reporting days, IQR 19.8-88.1\%). Post-EMI, most or all participants positively rated statements about the app and the EMI messages (Table 4), including that the messages made them feel better $(100 \%)$ and helped them to make the changes they wanted to make $(100 \%)$. In free text, they described having more confidence, awareness, and skills to manage their feelings, communicate with their partners, and engage in safer sex; feeling better about themselves; and/or practicing safer sex.

\section{Change in mental health and motivation to change behaviors}

Post-EMI vs. baseline, participants reported lower PHQ9 depressive symptom scores, higher confidence to change negative thoughts into positive thoughts (cognitive restructuring skill), and higher confidence to change their self-selected risk behavior (Table 5). At 3 months, participants' depressive symptom scores remained lower and confidence in ability to use cognitive restructuring remained higher, compared to baseline. Confidence to change behavior remained high and readiness to change behavior increased at 3 months, although differences from baseline were not significant. Importance of behavior change, which was high at baseline, increased post-EMI to the highest level and remained there at 3-month follow-up; differences between study phases were not significant.

\section{Change in sexual and reproductive bealth behaviors}

Participants reported decline in the frequency of sexual intercourse during the intervention and on 30-day recall at 3 -month follow-up, compared to the 30 days prior to baseline [median of 6 times at baseline (IQR 1.8-9.3), vs. 1 time during the intervention (IQR 1.0-6.5) and 2 times at follow-up (IQR 1.0-4.3)] (Table 5). Participants also reported decline in the proportion of condom-unprotected sex events by 3 months follow-up [median of $100 \%$ at baseline (IQR, 91.7-100.0), 100\% during the intervention (IQR, 0.0-100.0), and $0.0 \%$ at 3 months follow-up (IQR, 0.0-75.0)] (Table 5). Proportion of sex events with a new or casual partner did not change significantly over the study (Table 5).

From baseline to follow-up, contraceptive use changed very little. One participant reporting no birth control at baseline reported condoms for birth control at followup, another reported the depot medroxyprogesterone shot at baseline and condoms for birth control at followup, and two participants changed from one hormonal form of contraception to another (implant to shot, oral contraceptive pill to contraceptive vaginal ring). Proportion of participants using effective birth control who were using condoms consistently (with $100 \%$ of sex events) increased 
Table 5 Mental Health and motivation to change selected risk behavior-baseline, post-intervention, and 3-month follow-up

\begin{tabular}{|c|c|c|c|c|c|}
\hline Variable & $\begin{array}{l}\text { Baseline, } \\
\text { median (IQR) }\end{array}$ & \multicolumn{2}{|c|}{ Post-EMI } & \multicolumn{2}{|c|}{ 3-month follow-up } \\
\hline \multicolumn{6}{|l|}{ Mental health } \\
\hline $\begin{array}{l}\text { Depressive symptoms score } \\
(0-27)\end{array}$ & $12[9-18]$ & $10[5-12]$ & $Z=-2.559 ; P=0.010$ & $7[5-11]$ & $Z=-3.078 ; P=0.002$ \\
\hline \multicolumn{6}{|c|}{ Motivation to change selected risk behavior } \\
\hline Readiness (0-10) & $7(5.25-8.375)$ & $8[7-10]$ & $Z=-1.372 ; P=0.170$ & $10(7.5-10)$ & $Z=-1.881 ; P=0.060$ \\
\hline Confidence $(0-10)$ & $6[5-7]$ & $10(7.5-10)$ & $Z=-2.823 ; P=0.005$ & $9(6.5-10)$ & $Z=-1.747 ; P=0.081$ \\
\hline Number of sex events & $6.0(1.8-9.3)$ & $1.0(1.0-6.5)$ & $Z=-2.196 ; P=0.028$ & $2.0(1.0-4.3)$ & $Z=-1.963 ; P=0.050$ \\
\hline $\begin{array}{l}\text { Percent of condom-unprotected } \\
\text { sex events }\end{array}$ & $100.0(91.7-100.0)$ & $100.0(0.0-100.0)$ & $Z=-0.756 ; P=0.450$ & $0.0(0.0-75.0)$ & $Z=-2.585 ; P=0.010$ \\
\hline $\begin{array}{l}\text { Percent of sex events with new } \\
\text { or casual partner }\end{array}$ & $0.0(0.0-62.5)$ & $0.0(0.0-100.0)$ & $Z=-1.069 ; P=0.285$ & $0.0(0.0-25.0)$ & $Z=0.365 ; P=0.715$ \\
\hline
\end{tabular}

Results of Wilcoxon signed-rank test for related samples.

from $6.3 \%$ at baseline to $55.6 \%$ at 3 -month follow-up.

\section{Discussion}

Working collaboratively and iteratively with young women with depression, we developed an evidence-based counseling-plus-mHealth intervention to reduce risk for pregnancy and STI that reflected their opinions and needs. Findings from the subsequent pilot test provide evidence for feasibility and acceptability of the intervention. The intervention design offers a balance between intervention individualization and implementation considerations. Participants used and expressed appreciation for the opportunities for choice in the intervention to personalize the SRH risk behavior to change, the EMI message style and voice, the format of the booster session, and when to complete EMI surveys, yet the manualized counseling and pre-programmed EMI is practical for busy care settings and limits the training required for intervention administration.

Young women with depression in the pilot test rated the counseling highly, reflecting the counselors' application of the four central components of the MI Spirit: partnership; acceptance; compassion; and evocation (58). As seen in other research with adolescents and young adults (40), as well as with individuals who have psychiatric symptoms (69), EMI was a highly acceptable form of intervention. In general, participants were not annoyed by the report prompts or message frequency and app engagement remained high for the 4 weeks of the EMI. Our results on engagement compare favorably with previous research. In two studies of a counseling-plus-EMI for youth using cannabis frequently, participants responded to less than one-half of EMI momentary and daily prompts over the 2-week EMI $(36,40)$. In a study of an EMI for adults with psychotic disorder which prompted brief assessment and intervention up to 3 times a day (65), days of engagement with the EMI declined over 24 weeks from a high of mean 3.9 days in week 1 . In contrast, participants in our study engaged with the EMI on a median of 7 days a week for the in the $1^{\text {st }}$ week, declining only slightly (to median 5 days) by the $4^{\text {th }}$ week.

All or most of the participants found the messages helped them to feel better, use the skill taught in counseling, change their behaviors, and be safer, suggesting that the 
messaging approach holds promise for extending support for behavior change from the initial counseling session into daily life. In addition, we observed changes in intermediate outcomes targeted by the intervention. Participants reported increased confidence to reduce a personally-selected SRH risk behavior, befitting the MI-based counseling. Although not statistically significant differences, readiness to change and importance of changing the behavior were also higher post-intervention and at 3 months, compared to baseline. Participants also reported increased confidence to use cognitive restructuring, the skill taught in the counseling session and reinforced in natural life using an EMI, as well as decreased depressive symptoms post-intervention and at 3-month follow-up. Finally, although the study was not designed to assess efficacy, we also observed significant improvement in SRH risk behaviors 3 months after the intervention (less frequent sex and less condomunprotected sex than reported at baseline). Importantly, substantially more participants who were using effective contraception reported consistent condom use at followup. An appropriately powered randomized controlled trial is required to determine whether these changes can be attributed to MARSSI.

Our development and pilot-testing process had several strengths, including applying our team's experience with intervention development $(33,40)$, and with momentary assessment of affect and sexual behavior in depressed young women $(12,14)$; collaborating with members of the priority population; developing the intervention over successive iterations; and using qualitative and quantitative methods to gather data on feasibility and acceptability. The study did have several limitations. We used small samples of participants drawn from clinics affiliated with a single academic medical center, limiting the generalizability of the findings. We conducted many assessments, a burden which may have hampered enrollment and retention. We were not able to solicit feedback from participants who withdrew or were lost to follow-up to determine whether their experiences with study burden or other aspects of feasibility differed from those of participants who stayed in the study. Retained participants' feedback may have been subject to social desirability bias; we sought to reduce this form of bias by assuring confidentiality, using computer-based surveys, and offering balanced response options. The initial and booster counseling sessions exceeded our goals for duration, which may challenge implementation in non-research clinic settings. We are using the results of this research to streamline the counseling in an effort to shorten the session durations.

In conclusion, a counseling-plus-mHealth intervention that uses evidence-based motivational, affect regulatory, and cognitive-behavioral approaches to target the interplay between depression and sexual risk is feasible and acceptable to high-risk young women with depressive symptoms. MARSSI's design uses a comprehensive and personal counseling session to provide information, elicit motivation, develop an individualized behavior change plan, and teach cognitive-behavioral skills, while keeping this more resource-intense and in-person portion of the intervention to a single initial encounter. In using mobile technology for real-time self-monitoring and context-responsive intervention in daily life, the EMI component contributes to MARSSI's practicality and scalability and may enhance its efficacy.

\section{Acknowledgments}

The authors would like to acknowledge the young women who shared their knowledge and experiences and the clinical staff who referred patients for their assistance in realizing this study. Findings were presented in part at the American Public Health Association annual meeting, November 2017, Atlanta, GA, the Society for Adolescent Health and Medicine annual meeting, March 2019, Washington, DC, and the Society for Ambulatory Assessment biennial meeting, June 2019 Syracuse, NY.

Funding: This opportunity is made possible by Grant Number TP2AH000046 from the HHS Office of Adolescent Health as part of the Innovative Teen Pregnancy Prevention Programs ( $\left.\mathrm{iTP}_{3}\right)$ project. Contents are solely the responsibility of Boston Children's Hospital and do not necessarily represent the official views of the Department of Health and Human Services, the Office of Adolescent Health, or Texas A\&M University. This work was also supported by the Aerosmith Endowment Fund, Boston Children's Hospital.

\section{Footnote}

Conflicts of Interest: LAS serves as an unpaid editorial board member of mHealth from Mar. 2019 to Feb. 2021. The other authors have no conflicts of interest to declare.

Etbical Statement: The authors are accountable for all aspects of the work in ensuring that questions related to the accuracy or integrity of any part of the work are 
appropriately investigated and resolved.

Open Access Statement: This is an Open Access article distributed in accordance with the Creative Commons Attribution-NonCommercial-NoDerivs 4.0 International License (CC BY-NC-ND 4.0), which permits the noncommercial replication and distribution of the article with the strict proviso that no changes or edits are made and the original work is properly cited (including links to both the formal publication through the relevant DOI and the license). See: https://creativecommons.org/licenses/by-ncnd/4.0\%.

\section{References}

1. Hall KS, Kusunoki Y, Gatny H, et al. The risk of unintended pregnancy among young women with mental health symptoms. Soc Sci Med 2014;100:62-71.

2. James-Hawkins L, Denardo D, Blalock C, et al. Do depressive symptoms in male and female adolescents predict unintended births in emerging adulthood? Matern Child Health J 2014;18:2115-23.

3. Shrier LA, Harris SK, Beardslee WR. Temporal associations between depressive symptoms and selfreported sexually transmitted disease among adolescents. Arch Pediatr Adolesc Med 2002;156:599-606.

4. Lee SH, O'Riordan MA, Lazebnik R. Relationships among depressive symptoms, sexually transmitted infections, and pregnancy in African-American adolescent girls. J Pediatr Adolesc Gynecol 2009;22:19-23.

5. Stidham Hall K, Moreau C, Trussell J, et al. Young women's consistency of contraceptive use--does depression or stress matter? Contraception 2013;88:641-9.

6. Lehrer JA, Shrier LA, Gortmaker S, et al. Depressive symptoms as a longitudinal predictor of sexual risk behaviors among US middle and high school students. Pediatrics 2006;118:189-200.

7. Shrier LA, Aneja P, Rice PA, et al. Depression and STI risk within young, Chlamydia-infected, heterosexual dyads. J Adolesc Health 2009;45:63-9.

8. Fu H, Darroch JE, Haas T, et al. Contraceptive failure rates: new estimates from the 1995 National Survey of Family Growth. Fam Plann Perspect 1999;31:56-63.

9. Cooper ML, Agocha VB, Sheldon MS. A motivational perspective on risky behaviors: the role of personality and affect regulatory processes. J Pers 2000;68:1059-88.

10. Hessler DM, Katz LF. Brief report: Associations between emotional competence and adolescent risky behavior. J
Adolesc 2010;33:241-6.

11. Brown LK, Houck C, Lescano C, et al. Affect regulation and HIV risk among youth in therapeutic schools. AIDS Behav 2012;16:2272-8.

12. Mehta CM, Walls C, Blood EA, et al. Associations between affect, context, and sexual desire in depressed young women. J Sex Res 2014;51:577-85.

13. Kendall AD, Wilt J, Walls CE, et al. The social context of positive and negative affective states in depressed youth. J Soc Clin Psychol 2014;33:805-30.

14. Shrier LA, Feldman HA, Black SK, et al. Momentary affective states surrounding sexual intercourse in depressed adolescents and young adults. Arch Sex Behav 2012;41:1161-71.

15. Katz RC, Mills K, Singh NN, et al. Knowledge and attitudes about AIDS: A comparison of public high school students, incarcerated delinquents, and emotionally disturbed adolescents. J Youth Adolesc 1995;24:117-31.

16. Lopez V, Kopak A, Robillard A, et al. Pathways to sexual risk taking among female adolescent detainees. J Youth Adolesc 2011;40:945-57.

17. Shrier LA, Harris SK, Kurland M, et al. Substance use problems and associated psychiatric symptoms among adolescents in primary care. Pediatrics 2003;111:e699-705.

18. Cooper ML, Shapiro CM, Powers AM. Motivations for sex and risky sexual behavior among adolescents and young adults: a functional perspective. J Pers Soc Psychol 1998;75:1528-58.

19. Wingood GM, DiClemente RJ. Application of the theory of gender and power to examine HIV-related exposures, risk factors, and effective interventions for women. Health Educ Behav 2000;27:539-65.

20. DiClemente RJ, Wingood GM, Crosby RA, et al. A prospective study of psychological distress and sexual risk behavior among black adolescent females. Pediatrics 2001;108:E85.

21. Park J, Nordstrom SK, Weber KM, et al. Reproductive coercion - uncloaking an imbalance of social power. Am J Obstet Gynecol 2016;214:74-8.

22. Lehrer JA, Buka S, Gortmaker S, et al. Depressive symptomatology as a predictor of exposure to intimate partner violence among US female adolescents and young adults. Arch Pediatr Adolesc Med 2006;160:270-6.

23. Francis J, Malbon K, Braun-Courville D, et al. Ambivalence about pregnancy and its association with symptoms of depression in adolescent females initiating contraception. J Adolesc Health. 2015;56:44-51.

24. Centers for Disease Control and Prevention. Compendium 
of Evidence-Based HIV Prevention Interventions 2014. Available online: http://www.cdc.gov/hiv/topics/research/ prs/promising-evidence-interventions.htm\#completelist

25. Brown LK, Reynolds LA, Lourie KJ. A pilot HIV prevention program for adolescents in a psychiatric hospital. Psychiatr Serv 1997;48:531-3.

26. Thurstone C, Riggs PD, Klein C, et al. A one-session human immunodeficiency virus risk-reduction intervention in adolescents with psychiatric and substance use disorders. J Am Acad Child Adolesc Psychiatry 2007;46:1179-86.

27. Sterk CE, Theall KP, Elifson KW. The impact of emotional distress on HIV risk reduction among women. Subst Use Misuse 2006;41:157-73.

28. Brown LK, Nugent NR, Houck CD, et al. Safe Thinking and Affect Regulation (STAR): human immunodeficiency virus prevention in alternative/therapeutic schools. J Am Acad Child Adolesc Psychiatry 2011;50:1065-74.

29. Holden AEC, Shain RN, Miller WB, et al. The influence of depression on sexual risk reduction and STD infection in a controlled, randomized intervention trial. Sex Transm Dis 2008;35:898-904.

30. Sales JM, Lang DL, Hardin JW, et al. Efficacy of an HIV prevention program among African American female adolescents reporting high depressive symptomatology. J Womens Health (Larchmt) 2010;19:219-27.

31. Compton WM, Cottler LB, Ben-Abdallah A, et al. The effects of psychiatric comorbidity on response to an HIV prevention intervention. Drug Alcohol Depend 2000;58:247-57.

32. Brown JL, Sales JM, Swartzendruber AL, et al. Added benefits: reduced depressive symptom levels among AfricanAmerican female adolescents participating in an HIV prevention intervention. J Behav Med 2014;37:912-20.

33. Shrier LA, Ancheta R, Goodman E, et al. Randomized controlled trial of a safer sex intervention for high-risk adolescent girls. Arch Pediatr Adolesc Med 2001;155:73-9.

34. DiClemente RJ, Wingood GM, Harrington KF, et al. Efficacy of an HIV prevention intervention for African American adolescent girls: a randomized controlled trial. JAMA 2004;292:171-9.

35. Kauer SD, Reid SC, Crooke AH, et al. Self-monitoring using mobile phones in the early stages of adolescent depression: randomized controlled trial. J Med Internet Res 2012;14:e67.

36. Shrier LA, Rhoads A, Burke P, et al. Real-time, contextual intervention using mobile technology to reduce marijuana use among youth: a pilot study. Addict Behav 2014;39:173-80.
37. Blood EA, Shrier LA. The temporal relationship between momentary affective states and condom use in depressed adolescents. Arch Sex Behav 2013;42:1209-16.

38. Burns MN, Begale M, Duffecy J, et al. Harnessing context sensing to develop a mobile intervention for depression. J Med Internet Res 2011;13:e55.

39. Shrier LA, Burke PJ, Kells M, et al. Pilot randomized trial of MOMENT, a motivational counseling-plus-ecological momentary intervention to reduce marijuana use in youth. mHealth 2018;4:29.

40. Heron KE, Smyth JM. Ecological momentary interventions: incorporating mobile technology into psychosocial and health behaviour treatments. Br J Health Psychol 2010;15:1-39.

41. Pew Research Center. Teen, social media and technology overview 2018. Available online: https://www.pewinternet. org/2018/05/31/teens-social-media-technology-2018/

42. Pew Research Center. Mobile technology and home broadband 2019. Available online: http://www. pewinternet.org/2019/06/13/mobile-technology-andhome-broadband-2019/

43. Pew Research Center. Smartphone ownership is growing rapidly around the world, but not always equally 2019. Available online: https://www.pewresearch.org/ global/2019/02/05/digital-connectivity-growing-rapidlyin-emerging-economies/

44. Pew Research Center. Teens' social media habits and experiences. 2018. Available online: https://www. pewinternet.org/2018/11/28/teens-social-media-habitsand-experiences/

45. Shrier LA, Spalding A. "Just take a moment and breathe and think": young women with depression talk about the development of an Ecological Momentary Intervention to reduce their sexual risk. J Pediatr Adolesc Gynecol 2017;30:116-22.

46. Whittaker R, McRobbie H, Bullen C, et al. Mobile phonebased interventions for smoking cessation. Cochrane Database Syst Rev 2016;4:CD006611.

47. Cunningham RM, Chermack ST, Zimmerman MA, et al. Brief motivational interviewing intervention for peer violence and alcohol use in teens: one-year follow-up. Pediatrics 2012;129:1083-90.

48. Brottman M. Whereof one cannot speak: conducting psychoanalysis online. Psychoanal Rev 2012;99:19-34.

49. Shrier LA, Rhoads AM, Fredette ME, et al. "Counselor in your pocket": youth and provider perspectives on a mobile motivational intervention for marijuana use. Subst Use Misuse 2014;49:134-44. 
50. Kirby D. BDI logic models: a useful tool for designing, strengthening, and evaluating programs to reduce adolescent sexual risk-taking, pregnancy, HIV, and other STDs. Scotts Valley, CA: ETR Associates, 2004.

51. Fedorowicz AR, Hellerstedt WL, Schreiner PJ, et al. Associations of adolescent hopelessness and self-worth with pregnancy attempts and pregnancy desire. Am J Public Health 2014;104:e133-e40.

52. Seth P, Raiji PT, DiClemente RJ, et al. Psychological distress as a correlate of a biologically confirmed STI, risky sexual practices, self-efficacy and communication with male sex partners in African-American female adolescents. Psychol Health Med 2009;14:291-300.

53. Carvajal DN, Ghazarian SR, Crowne SS, et al. Is depression associated with contraceptive motivations, intentions, and use among a sample of low-income Latinas? Womens Health Issues 2014;24:e105-e113.

54. Cooper ML, Barber LL, Zhaoyang R, et al. Motivational pursuits in the context of human sexual relationships. J Pers 2011;79:1333-68.

55. Shrier LA, Walls C, Lops C, et al. Substance use, sexual intercourse, and condom nonuse among depressed adolescents and young adults. J Adolesc Health 2012;50:264-70.

56. Burke PJ, Katz-Wise SL, Spalding A, et al. Intimate relationships and sexual behavior in young women with depression. J Adolesc Health 2018;63:429-34.

57. Treadway MT, Bossaller NA, Shelton RC, et al. Effortbased decision-making in major depressive disorder: a translational model of motivational anhedonia. J Abnorm Psychol 2012;121:553-8.

58. Miller WR, Rollnick S. Motivational interviewing: Helping people change (3rd edition). New York, NY, US: Guilford Press, 2013.

59. Kelsey M. Safer Sex Intervention Short-Term Impacts. Teen Pregnancy Prevention Replication Study. Cambridge, MA: Abt Associates, 2015.

60. Kiene SM, Bateganya MH, Lule H, et al. The Effect of motivational interviewing-based counseling during

doi: $10.21037 /$ mhealth.2019.11.05

Cite this article as: Shrier LA, Burke PJ, Parker S, Edwards R, Jonestrask C, Pluhar E, Harris SK. Development and pilot testing of a counseling-plus-mHealth intervention to reduce risk for pregnancy and sexually transmitted infection in young women with depression. mHealth 2020;6:17. outpatient provider initiated HIV testing on highrisk sexual behavior in rural Uganda. AIDS Behav 2016;20:1928-36.

61. Klein JB, Jacobs RH, Reinecke MA. Cognitive-behavioral therapy for adolescent depression: A meta-analytic investigation of changes in effect-size estimates. J Am Acad Child Adolesc Psychiatry 2007;46:1403-13.

62. Reinecke MA, Jacobs RH. Cognitive-behavioral therapy for depression. In: Dulcan MK, editor. Dulcan's Textbook of Child and Adolescent Psychiatry. Arlington, VA US: American Psychiatric Publishing, Inc., 2010:907-14.

63. Kroenke K, Spitzer RL, Williams JBW. The PHQ9: validity of a brief depression severity measure. J Gen Intern Med 2001;16:606-13.

64. Carey MP, Carey KB, Maisto SA, et al. Assessing sexual risk behaviour with the Timeline Followback (TLFB) approach: continued development and psychometric evaluation with psychiatric outpatients. Int J STD AIDS 2001;12:365-75.

65. Ben-Zeev D, Scherer EA, Gottlieb JD, et al. mHealth for schizophrenia: patient engagement with a mobile phone intervention following hospital discharge. JMIR Ment Health 2016;3:e34.

66. Chesney MA, Neilands TB, Chambers DB, et al. A validity and reliability study of the coping self-efficacy scale. Br J Health Psychol 2006;11:421-37.

67. Pett MA.Nonparametric Statistics for Health Care Research: Statistics for Small Samples and Unusual Distributions, 2nd Edition. Thousand Oaks, CA: Sage Publications, Inc., 2015.

68. Manea L, Gilbody S, McMillan D. Optimal cutoff score for diagnosing depression with the Patient Health Questionnaire (PHQ-9): A meta-analysis. CMAJ 2012;184:E191-E196.

69. Wenze SJ, Armey MF, Miller IW. Feasibility and acceptability of a mobile intervention to improve treatment adherence in bipolar disorder: a pilot study. Behav Modif 2014;38:497-515. 